\title{
Deterioration of Health-Related Quality of Life After Withdrawal of Risankizumab Treatment in Patients with Moderate-to-Severe Plaque Psoriasis: A Machine Learning Predictive Model
}

\author{
Kim A. Papp (D) - Ahmed M. Soliman · Nicolae Done · \\ Christopher Carley · Esteban Lemus Wirtz · Luis Puig
}

Received: March 26, 2021 / Accepted: May 6, 2021 / Published online: May 21, 2021

(C) The Author(s) 2021

\begin{abstract}
Introduction: Risankizumab has demonstrated efficacy in treating moderate-to-severe psoriasis. The phase-3 IMMhance trial (NCT02672852) examined the effect of continuing versus withdrawing from risankizumab treatment on psoriasis severity, including the Psoriasis Area and Severity Index (PASI) and static Physician Global Assessment (sPGA). However, the effect of withdrawal on health-related quality of life (HRQL) was not assessed. Therefore, this study was conducted to evaluate the impact of risankizumab withdrawal on HRQL measured by the Dermatology Life Quality Index (DLQI). Because DLQI was not measured beyond week
\end{abstract}

Supplementary Information The online version contains supplementary material available at https:// doi.org/10.1007/s13555-021-00550-8.

\section{K. A. Papp (ه)}

K Papp Clinical Research and Probity Medical

Research, Waterloo, ON, Canada

e-mail: kapapp@probitymedical.com

\section{A. M. Soliman}

AbbVie, Inc., North Chicago, IL, USA

N. Done · C. Carley · E. Lemus Wirtz

Analysis Group, Inc., Boston, MA, USA

\section{Puig}

Department of Dermatology, Hospital de la Santa

Creu i Sant Pau, Universitat Autònoma de

Barcelona, Barcelona, Spain
16 in IMMhance, a machine learning predictive model for DLQI was developed.

Methods: A machine learning model for DLQI was fitted using repeated measures data from three phase-3 trials (NCT02684370, NCT02684357, NCT02694523) (pooled $N=1602$ ). An elastic-net algorithm performed automated variable selection among candidate predictors including concurrent PASI and sPGA, demographics, and interaction terms. The machine learning model was used to predict DLQI at weeks 28-104 of IMMhance among patients re-randomized to continue $(N=111)$ or withdraw from $(N=225)$ risankizumab after achieving response $(\mathrm{sPGA}=0 / 1)$ at week 28 .

Results: The machine learning predictive model demonstrated good statistical fit during tenfold cross-validation and external validation against observed DLQI at weeks $0-16$ of IMMhance $(N=507)$. Predicted improvements in DLQI from baseline were lower in the withdrawal versus the continuation cohort (mean DLQI change at week $104,-5.9$ versus -11.5 , difference $[95 \% \mathrm{CI}]=5.6[4.1,7.3])$. Predicted DLQI deteriorated more extensively than PASI $(49.7 \%$ versus $36.4 \%)$ after treatment withdrawal.

Conclusions: The predicted DLQI score deteriorated more rapidly after risankizumab withdrawal than the PASI score, an objective measure of disease. These findings suggest that the deterioration in HRQL reflects more substantial impacts after risankizumab 
discontinuation than those measured by PASI only.

Keywords: DLQI; Machine learning; PASI; Psoriasis; Quality of life; Risankizumab

\section{Key Summary Points}

Why carry out this study?

Although the efficacy of risankizumab in the treatment of moderate-to-severe psoriasis has been demonstrated in a recent phase 3 clinical trial, the impact of its withdrawal on patients' health-related quality of life (HRQL) has not yet been assessed.

This study was conducted to gain insight regarding the impact of risankizumab withdrawal on HRQL among patients with moderate-to-severe psoriasis from IMMhance using the Dermatology Life Quality Index (DLQI); however, due to the absence of DLQI collected beyond week 16 in clinical trials, a machine learning predictive model was developed to assess the DLQI among patients with moderateto-severe psoriasis.

\section{What was learned from the study?}

The DLQI scores calculated using the machine learning predictive model showed that patients who withdrew from risankizumab experienced more extensive deterioration in their HRQL compared with patients who continuously remained on treatment.

When compared with the Psoriasis Area and Severity Index (PASI), a commonly used objective measure of clinical severity, the DLQI score showed a larger relative decline, suggesting that this measure of HRQL is affected by risankizumab withdrawal more extensively than clinical measures would suggest.

\section{DIGITAL FEATURES}

This article is published with digital features, including a summary slide, to facilitate understanding of the article. To view digital features for this article, go to https://doi.org/10.6084/ m9.figshare.14541864.

\section{INTRODUCTION}

Plaque psoriasis is a chronic inflammatory skin condition, characterized by pruritic and scaly lesions (plaques) throughout the patient's body [1]. Patients with plaque psoriasis experience debilitating pain, stigmatization [2], and psychosocial impairments $[3,4]$ due to the appearance of lesions, and are susceptible to multiple comorbidities (e.g., metabolic syndrome, cardiovascular disease) [5]. This has resulted in a substantial decrease in patients' health-related quality of life (HRQL) [6-9], which typically correlates with disease severity (e.g., mild, moderate, severe) [5].

Although there is no cure for psoriasis, treatments are available to assist in providing sustained clearance of lesions, particularly among patients with moderate-to-severe plaque psoriasis [10]. Several clinician-reported outcome measures are used to evaluate the magnitude of the disease burden and the effectiveness of therapies in clinical studies and real-world practice [11]. The Psoriasis Area and Severity Index (PASI) is a commonly used instrument for measuring psoriasis severity and evaluating treatment response. PASI scores measure psoriasis symptom involvement of four body sections: head, upper extremities, trunk, and lower extremities [12]. The static Physician Global Assessment (sPGA) is a widely used, clinician-reported instrument for measuring psoriasis severity based on induration, scaling, and erythema, with scores from 0 (clear) to 5 (severe) [13]. Although clinician-reported instruments can provide objective assessments of symptom control, assessments of treatment effectiveness must also include the patient perspective, particularly during clinical trials used to inform treatment selection and optimization in real-world practice. This requires the use of 
instruments that incorporate patient-reported outcomes (PROs) [14, 15]. The Dermatology Quality Index (DLQI) is a PRO tool used to assess HRQL across six domains (patient's perception of symptoms and feelings, daily activities, leisure, work and school, interpersonal relationships, and treatment) [16].

Risankizumab is a fully humanized monoclonal antibody with a high affinity for the p19 component of interleukin (IL)-23, a cytokine that plays a key role in the pathogenesis of psoriasis [17]. Recently, data from four large, multicenter, phase 3 randomized controlled trials, including UltIMMa-1 (NCT02684370), UltIMMa-2 (NCT02684357), IMMvent (NCT02694523), and IMMhance (NCT02672852), showed significantly greater efficacy with risankizumab versus placebo [18-20], ustekinumab [19], and adalimumab [20] as measured by relative improvements from baseline in PASI and DLQI. The IMMhance trial also investigated the impact of randomized withdrawal from risankizumab among patients who achieved an initial response at week 28 [18]. Data from IMMhance showed that continuous treatment provided significantly better long-term disease control than withdrawal (at week 52: $87 \%$ versus 61\%, $p<0.001$ ) [21]. However, in IMMhance, DLQI was only collected through week 16 , and thus the corresponding impact of treatment withdrawal on HRQL could not be directly assessed. To circumvent the limitation of outcome availability only for a limited number of patient visits, the current study assessed the deterioration of HRQL following risankizumab treatment withdrawal using a machine learning predictive model to assess DLQI among patients in the IMMhance trial.

\section{METHODS}

\section{Data Sources}

The data used in this study were from four phase-3 randomized, double-blind controlled, trials (UltIMMa-1, UltIMMa-2, IMMvent, and IMMhance) of risankizumab for the treatment of moderate-to-severe plaque psoriasis (Fig. 1).

\section{UltIMMa-1 and UltIMMa-2 Trials}

Patients in the UltIMMa-1 and UltIMMa-2 trials were randomized 3:1:1 at baseline to risankizu$\mathrm{mab}$ (150 mg at weeks 0 and 4 followed by every 12 weeks), ustekinumab ( 45 or $90 \mathrm{mg}$ at weeks 0 and 4 followed by every 12 weeks), or placebo (weeks 0 and 4 followed by cross-over to risankizumab 150 mg every 12 weeks after an initial 16-week double-blind period)[19]. DLQI was collected at weeks $0,12,16$, and 52. Candidate predictor variables were collected at all visits through week 52.

\section{IMMvent Trial}

Patients in the IMMvent trial were randomized $1: 1$ at baseline to risankizumab $(150 \mathrm{mg}$ at weeks 0 and 4 followed by every 12 weeks) or adalimumab (90 mg at week 0 , followed by $40 \mathrm{mg}$ at week 1 and every 2 weeks thereafter)[20]. At week 16, patients initially randomized to adalimumab were switched to risankizumab if $<$ PASI50, remained on adalimumab if $\geq$ PASI90, or were re-randomized 1:1 to either risankizumab or adalimumab if $\geq$ PASI50 and $<$ PASI90. DLQI was collected at weeks 0,16 , and 44 . Candidate predictor variables were collected at all visits through week 44.

\section{IMMhance Trial}

Patients in the IMMhance trial were randomized 4:1 at baseline to risankizumab $150 \mathrm{mg}$ or placebo [18]. Patients received a dose of study medication at week 0 , week 4 , and every 12 weeks thereafter. At week 16, patients randomized to placebo started receiving active treatment with risankizumab $150 \mathrm{mg}$ every 12 weeks and continued until the end of the treatment period. At week 28, patients randomized to risankizumab who achieved an sPGA score of 0 or 1 were re-randomized to either risankizumab or placebo. Starting from week 32, week 28 responders who experienced relapse (sPGA $\geq 3$ ) were switched to open-label risankizumab $150 \mathrm{mg}$ every 12 weeks. DLQI was collected at weeks 0,12 , and 16. Candidate predictor variables were collected at all visits through week 104. 


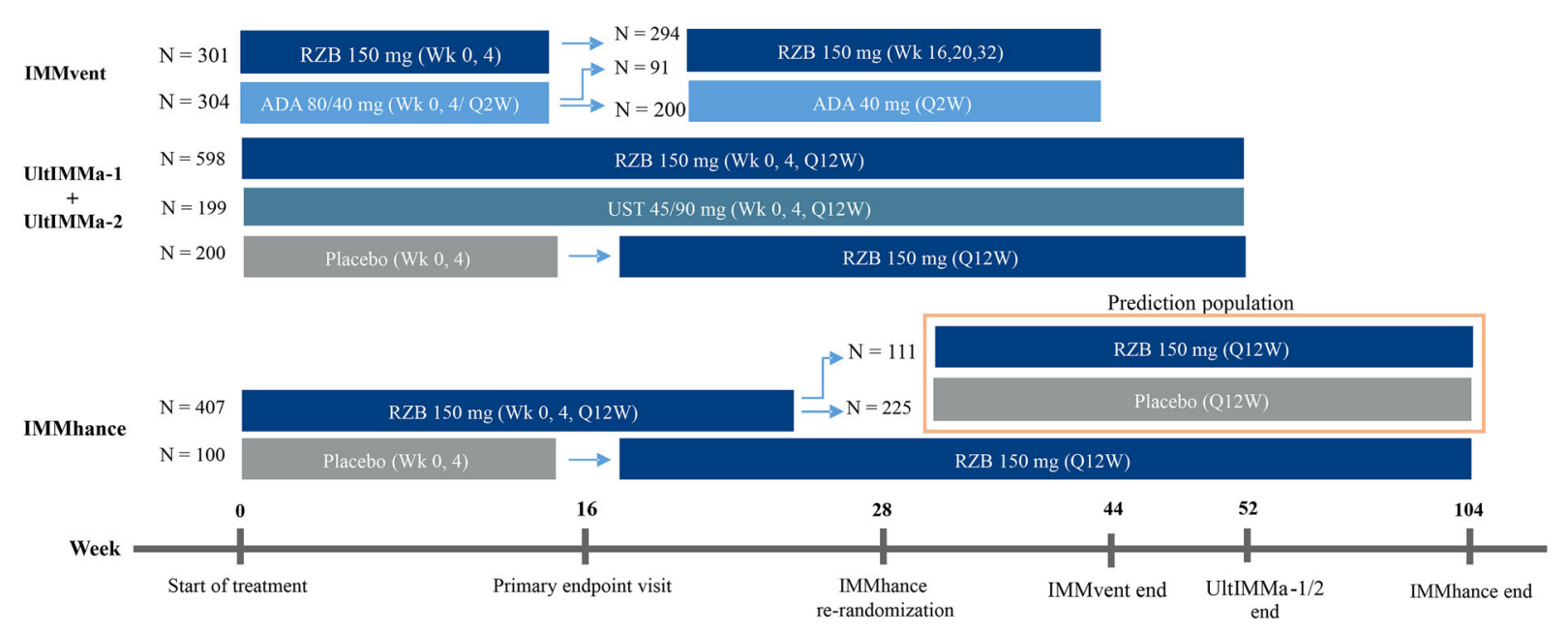

Fig. 1 Data sources and patient flowchart in the clinical trials included in the analysis. At week 28 of IMMhance, patients originally randomized to risankizumab who achieved a sPGA score of 0 or 1 were re-randomized to either risankizumab or placebo. Starting from week 32, week 28 responders who experienced relapse $($ sPGA $\geq 3$ )

\section{Inclusion Criteria}

Model development included all patients in the intention-to-treat populations of UltIMMa-1, UltIMMa-2, and IMMvent who had visits with concurrently available DLQI and predictor variable measurements. External model validation included all patients in the intention-totreat population of the IMMhance trial who had visits with concurrently available DLQI and predictor variable measurements at weeks 0,12 , or 16. In the score prediction phase, DLQI scores were predicted among patients originally randomized to risankizumab in IMMhance who achieved sPGA of 0 or 1 at week 28 and were consequently re-randomized to continue risankizumab or switch to placebo. The treatment cohort was defined according to the intentionto-treat re-randomization group. DLQI scores were predicted for patient visits with concurrently available predictor variable measurements.

\section{Compliance with Ethics Guidelines}

This article is based on previously conducted studies and does not contain any new studies were switched to open-label risankizumab $150 \mathrm{mg}$. $A D A$ adalimumab, $Q 2 W$ every 2 weeks, $Q 12 W$ every 12 weeks, $R Z B$ risankizumab, UST ustekinumab, $W k$ week

with human participants performed by any of the authors. The trials included in this study were approved by each site's institutional review board and were performed in accordance with the Helsinki Declaration of 1964, and its later amendments.

\section{Outcome Measure and Candidate Predictors}

The outcome of interest was the total DLQI score, which served as the dependent variable in the predictive regression models.

Candidate predictors including the PASI total score, PASI body section subscores, and the sPGA score were considered for inclusion in the prediction model of DLQI as concurrent measures of psoriasis severity. Baseline candidate predictors included demographics (age, sex, and race), weight, duration of psoriasis, and history of use of systemic biologic therapy. Additionally, several squared terms (age ${ }^{2}$, weight ${ }^{2}$, disease duration ${ }^{2}$, sPGA ${ }^{2}$, total PASI $^{2}$ ) and interaction terms (PASI $\times$ age, PASI $\times$ weight, PASI $\times$ sex, $\quad$ sPGA $\times$ age, sPGA $\times$ weight, sPGA $\times$ sex) were considered as candidate predictors. 


\section{Baseline Characteristics}

Baseline characteristics were summarized for the intention-to-treat populations in UltIMMa1, UltIMMa-2, IMMvent, and IMMhance, and for each re-randomized cohort in IMMhance. Patient characteristics assessed included DLQI and all candidate predictors. Means and standard deviations were summarized for continuous variables; counts and percentages were summarized for categorical variables. Statistical comparisons between re-randomized cohorts in the IMMhance trial were conducted using oneway analysis of variance for continuous variables and $\chi^{2}$ tests for dichotomous variables.

\section{Model Development and Validation}

Repeated-measures linear regression models for DLQI score prediction were developed with pooled data from UltIMMa-1, UltIMMa-2, and IMMvent, using cross-validation to prevent model overfitting. In addition to several model specifications with progressively expanded predictor subsets, machine learning algorithms including elastic net and least absolute shrinkage and selection operator (LASSO) regression were used to automate variable selection from among the full set of candidate predictors. The best-fitting models were further validated in the IMMhance population using all patient visits with concurrently available DLQI scores (at weeks 0,12 , and 16) and predictor variables. The predictive performance of all models was assessed using the cross-validated mean absolute error (MAE) and the coefficient of determination $\left(R^{2}\right)$.

\section{DLQI Prediction and Calculation of DLQI and PASI Deterioration}

Longitudinal analyses of predicted DLQI scores were conducted to examine the deterioration of HRQL associated with risankizumab treatment withdrawal after achievement of response in the IMMhance trial. The treatment cohort [risankizumab versus placebo, (i.e., withdrawal)] was defined according to intention-to-treat re-randomization groups. For each study visit in part
B (weeks $28+$ ) of the IMMhance trial, the mean change in DLQI score from baseline was modeled in each re-randomization group based on the difference between DLQI scores predicted at the visit versus the score observed at baseline. A bootstrapping approach was used to obtain 95\% confidence intervals (CIs) around the mean change from baseline in DLQI in each re-randomization group and around the betweengroup difference at each visit. Percent maintenance of score improvement from baseline to week 28 was assessed for predicted DLQI scores and observed PASI in each re-randomization group. For patients who switched to open-label risankizumab because of relapse, or who were lost to follow-up, last observation carried forward (LOCF) was used to impute PASI and sPGA scores at subsequent visits. Additional methodological details are provided in Appendix 1 of the electronic supplementary material.

\section{RESULTS}

\section{Baseline Characteristics}

A total of 2109 subjects were included in the samples used for model training and validation (UltIMMa-1 and UltIMMa-2: 997; IMMvent: 605; IMMhance: 507), and 336 subjects were included in the sample used for DLQI prediction (IMMhance re-randomized placebo: 225; risankizumab: 111). Baseline characteristics were well balanced across the intention-to-treat populations of the clinical trials used in the model development and validation phase (Table 1). The mean age across cohorts ranged between 46.2 and 49.3 years, and between $25.2 \%$ and $30.7 \%$ of patients were female. The mean DLQI score ranged between 13.0 and 13.7, and the total PASI score ranged between 19.7 and 20.2. Similarly, baseline characteristics were balanced across the re-randomized cohorts used for longitudinal DLQI score predictions.

\section{Model Development and Validation}

The prediction models for DLQI demonstrated significant, positive associations between DLQI 
Table 1 Baseline characteristics for patients whose data were used in the study

\begin{tabular}{|c|c|c|c|c|c|c|}
\hline & \multicolumn{3}{|c|}{ Sample used for model training and validation } & \multicolumn{3}{|c|}{$\begin{array}{l}\text { Sample used for DLQI } \\
\text { prediction (IMMhance re- } \\
\text { randomized) }\end{array}$} \\
\hline & $\begin{array}{l}\text { UltIMMa-1 \& } \\
\text { UltIMMa-2 } \\
N=997\end{array}$ & $\begin{array}{l}\text { IMMvent } \\
N=605\end{array}$ & $\begin{array}{l}\text { IMMhance } \\
N=507\end{array}$ & $\begin{array}{l}\text { PBO } \\
\text { patients } \\
N=225\end{array}$ & $\begin{array}{l}\text { RZB } \\
\text { patients } \\
N=111\end{array}$ & $\begin{array}{l}p- \\
\text { value }\end{array}$ \\
\hline \multicolumn{7}{|c|}{ Demographics and clinical characteristics } \\
\hline Age (years), mean $(S D)$ & $47.4 \pm 13.7$ & $46.2 \pm 13.5$ & $49.2 \pm 13.3$ & $49.3 \pm 13.1$ & $48.2 \pm 13.4$ & 0.457 \\
\hline Female, $n(\%)$ & $300(30.1 \%)$ & $183(30.2 \%)$ & $151(29.8 \%)$ & $69(30.7 \%)$ & $28(25.2 \%)$ & 0.301 \\
\hline White race, $n(\%)$ & $778(78.0 \%)$ & $508(84.0 \%)$ & $402(79.3 \%)$ & $177(78.7 \%)$ & $82(73.9 \%)$ & 0.326 \\
\hline Weight $(\mathrm{kg})$, mean $(\mathrm{SD})$ & $90.1 \pm 21.9$ & $90.1 \pm 23.9$ & $92.0 \pm 22.9$ & $91.0 \pm 22.2$ & $91.3 \pm 23.7$ & 0.897 \\
\hline $\begin{array}{l}\text { Duration of psoriasis (years), } \\
\text { mean (SD) }\end{array}$ & $18.1 \pm 12.5$ & $18.2 \pm 12.3$ & $19.9 \pm 13.0$ & $20.4 \pm 13.4$ & $19.4 \pm 13.1$ & 0.505 \\
\hline $\begin{array}{l}\text { Prior biologic systemic therapy, } \\
n(\%)\end{array}$ & $377(37.8 \%)$ & $229(37.9 \%)$ & $281(55.4 \%)$ & $125(55.6 \%)$ & $57(51.4 \%)$ & 0.467 \\
\hline \multicolumn{7}{|c|}{ Measure of disease severity, mean (SD) } \\
\hline DLQI & $13.0 \pm 7.0$ & $13.7 \pm 7.2$ & $13.4 \pm 7.1$ & $13.0 \pm 7.2$ & $13.0 \pm 6.9$ & 0.992 \\
\hline sPGA & $3.2 \pm 0.4$ & $3.2 \pm 0.4$ & $3.2 \pm 0.4$ & $3.2 \pm 0.4$ & $3.2 \pm 0.4$ & 0.302 \\
\hline \multicolumn{7}{|l|}{ PASI score } \\
\hline Total PASI score & $20.1 \pm 7.4$ & $19.8 \pm 7.5$ & $20.2 \pm 8.1$ & $19.7 \pm 7.3$ & $20.2 \pm 8.9$ & 0.526 \\
\hline \multicolumn{7}{|l|}{ Subtotal (area score $\times$ lesion score) } \\
\hline Head & $16.3 \pm 11.9$ & $16.9 \pm 11.8$ & $17.0 \pm 11.2$ & $16.7 \pm 10.3$ & $18.8 \pm 13.1$ & 0.113 \\
\hline Trunk & $18.1 \pm 10.3$ & $17.9 \pm 9.8$ & $18.5 \pm 10.7$ & $18.2 \pm 9.5$ & $18.5 \pm 11.8$ & 0.759 \\
\hline Upper extremities & $19.5 \pm 9.1$ & $18.5 \pm 9.1$ & $19.4 \pm 9.1$ & $19.3 \pm 9.0$ & $18.9 \pm 9.0$ & 0.724 \\
\hline Lower extremities & $22.9 \pm 9.7$ & $22.7 \pm 10.1$ & $22.6 \pm 10.5$ & $21.7 \pm 9.7$ & $22.6 \pm 11.4$ & 0.485 \\
\hline
\end{tabular}

DLQI Dermatology Life Quality Index, PASI Psoriasis Area and Severity Index, PBO placebo, RZB risankizumab, $S D$ standard deviation, $s P G A$ static Physician's Global Assessment

and PASI across all specifications tested (Supplemental Table S1). The saturated model and elastic-net selected model demonstrated the best predictive performance out of the models tested, as measured by $R^{2}$ and MAE. Due to the high predictive performance and relative parsimony, the elastic net model was chosen for the prediction of longitudinal DLQI scores among re-randomized patients in part B of the IMMhance trial.

The predicted DLQI scores among patients who were re-randomized at week 28 in the
IMMhance trial mirrored the observed DLQI scores closely at weeks 0,12 , and 16 , further demonstrating the high out-of-sample predictive performance of the elastic net model (Fig. 2a). Moreover, the predicted DLQI scores closely mirrored the observed scores for patients in the UltIMMa-1/2 and IMMvent trials who achieved PASI100 response at week 16 but then lost this response at week 52 or 44 (Fig. 2b), confirming the high predictive performance of the elastic net model among patients who 

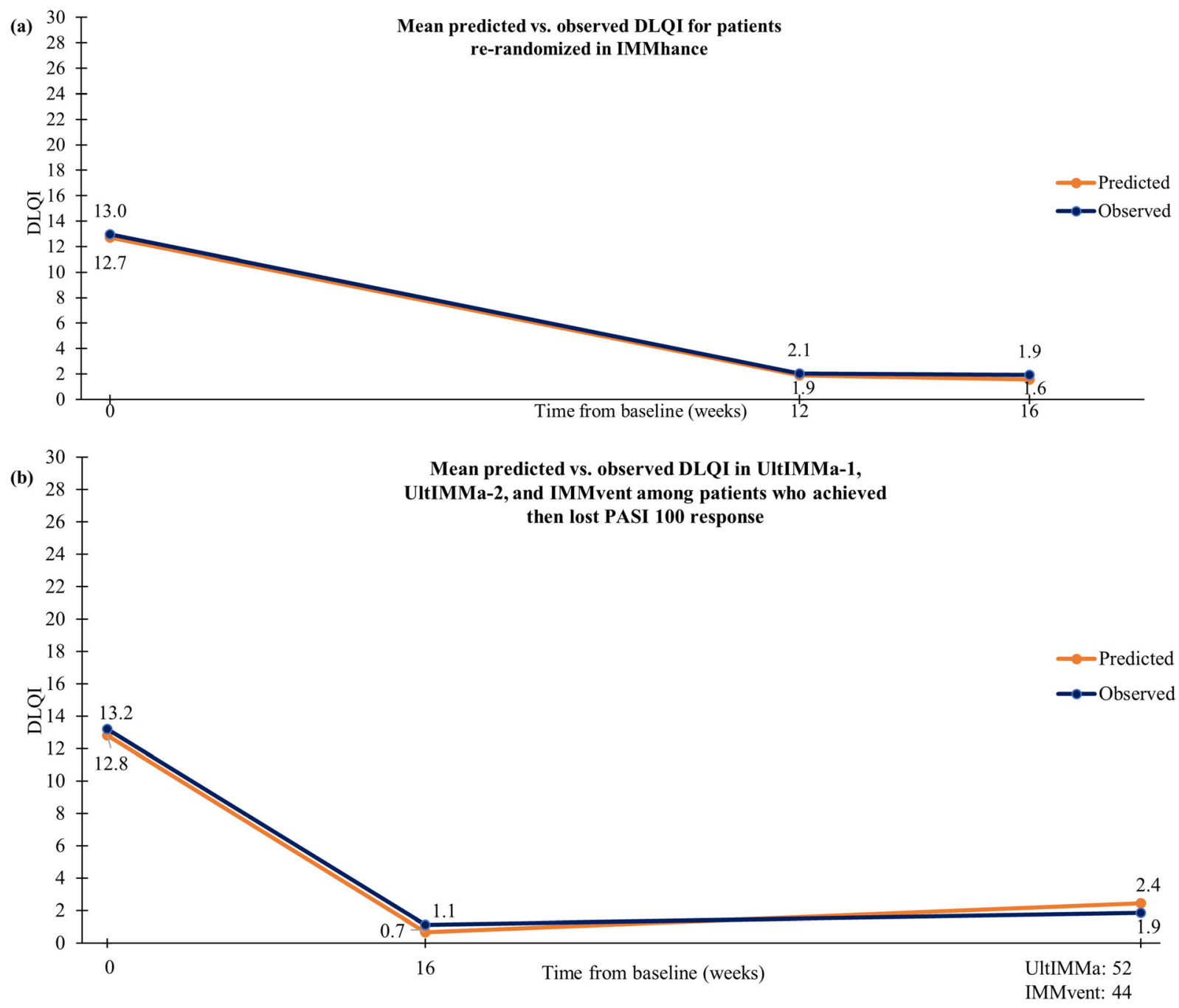

Fig. 2 a Mean predicted versus observed DLQI scores for weeks $0-16$ among patients re-randomized at week 28 in the IMMhance trial; $\mathbf{b}$ mean predicted versus observed DLQI scores in the UltIMMa-1, UltIMMa-2, and IMMvent trials among patients who achieved PASI 100

respond to therapy and then experience a deterioration of response.

\section{Predicted Change in DLQI}

Analysis of predicted DLQI scores using the elastic net model revealed significantly greater deterioration in the cohort re-randomized to placebo relative to the cohort re-randomized to continue risankizumab treatment (Fig. 3a). There was a statistically significant difference in response at week 16 and then lost PASI 100 response at week 44 or 52. DLQI Dermatology Life Quality Index, PASI Psoriasis Area and Severity Index

the change in DLQI from baseline between cohorts at week 52 [difference-in-difference $=1.7 ; 95 \% \mathrm{CI}=(0.2,3.4)]$. Beyond week 52 , increasingly larger mean changes in DLQI scores were observed in the withdrawal cohort relative to the risankizumab cohort. These differences were significant at each visit beyond week 52. Raw predicted DLQI scores among rerandomized patients are shown in Supplemental Fig. S1. 

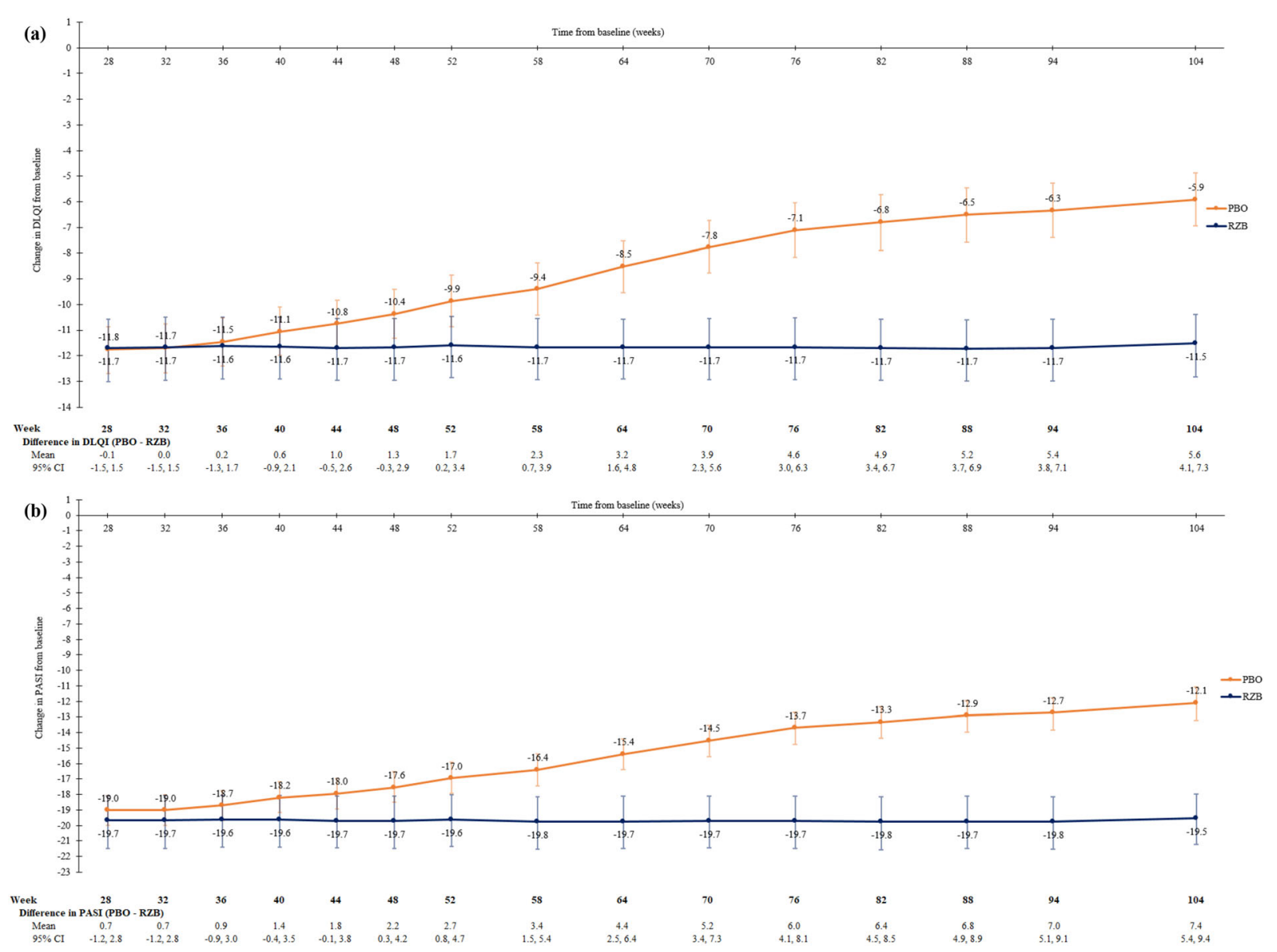

Fig. 3 a Change in predicted DLQI from baseline among re-randomized patients in the IMMhance trial. DLQI scores were predicted among patients originally randomized to risankizumab who were consequently re-randomized to continue risankizumab or switch to placebo in part B (weeks $28+$ ) of the IMMhance trial. An LOCF method was used to carry forward measurements for visits with missing measures; $\mathbf{b}$ change in PASI from baseline among re-randomized patients in the IMMhance trial.

\section{Maintenance of DLQI and PASI Score Improvement}

The cohort re-randomized to placebo showed deterioration in observed PASI scores and predicted DLQI scores over time, while the cohort re-randomized to continue risankizumab treatment had near-complete maintenance of both scores (Fig. 3). Predicted improvements in DLQI from baseline were lower in the withdrawal versus the continuation cohort [mean DLQI
PASI scores were assessed among patients originally randomized to risankizumab who were consequently rerandomized to continue risankizumab or switch to placebo in part B (weeks $28+$ ) of the IMMhance trial. An LOCF method was used to carry forward measurements for visits with missing measures. $C I$ confidence interval, $D L Q I$ Dermatology Life Quality Index, $L O C F$ last observation carried forward, $P B O$ placebo, $R Z B$ risankizumab

change at week $104,-5.9$ versus -11.5 , difference, 95\% CI $=5.6 \quad(4.1,7.3)$ ] (Fig. 3a). For patients in the withdrawal cohort, the DLQI score improvement achieved from baseline to week 28 deteriorated more rapidly after withdrawal compared with the deterioration in PASI score improvement (Fig. 4). At the end of follow-up (week 104), DLQI score improvement from baseline to week 28 had deteriorated by 49.7\%; PASI score improvement at week 28 deteriorated by $36.4 \%$ by week 104 . The 


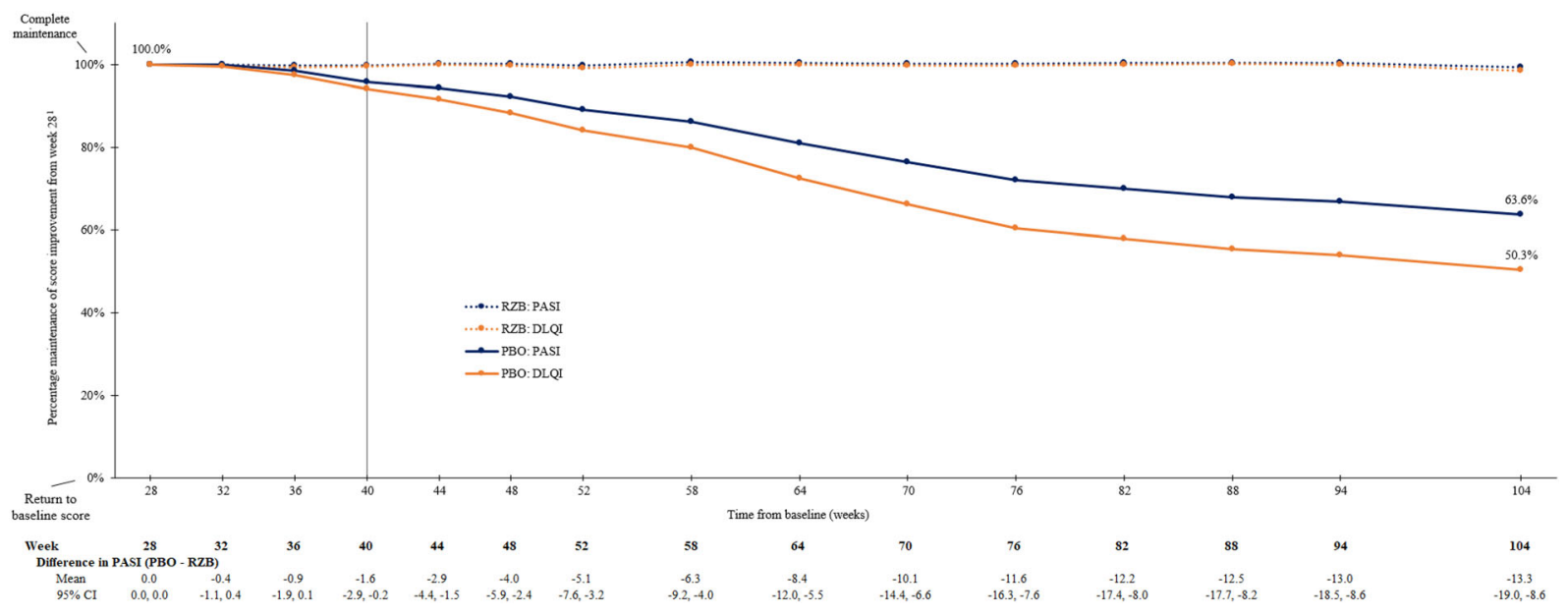

Fig. 4 Maintenance of DLQI and PASI score improvement following treatment re-randomization in the IMMhance trial. Maintenance of score improvement from week 28 was calculated as $100 \%-[100 \% \times$ (change in score from baseline to week 28 - change in score from baseline to week $x$ )/(change in score from baseline to week 28)]. Maintenance of $100 \%$ indicates complete maintenance of week 28 improvement; maintenance of $0 \%$

difference in deterioration of DLQI versus PASI among patients who withdrew from risankizumab was statistically significantly different by week 40 [difference $(95 \% \mathrm{CI})=-1.6 \%(-2.9 \%$, $-0.2 \%)$, and in all weeks thereafter (Fig. 4). Changes in sPGA from baseline are shown in Supplemental Figure S2.

\section{DISCUSSION}

Achieving skin clearance is a meaningful improvement to patients with moderate-tosevere psoriasis [22]. Due to the chronic nature of the disease, patients with psoriasis require ongoing treatment to adequately manage symptoms [23]. Although guidelines recommend continuous therapy for patients with psoriasis who are treated with biologic agents $[24,25]$, gaps in treatment are common in clinical practice $[26,27]$. Reasons for treatment interruption vary but can include lack of meaningful improvement, adverse events, and/ or patient preference to avoid continuous treatment $[28,29]$. The present study demonstrated that the predicted HRQL worsened at a indicates a return to baseline score. The vertical line at week 40 indicates the first visit when the percent deterioration difference in DLQI versus PASI becomes statistically significant. DLQI Dermatology Life Quality Index, LOCF last observation carried forward, PASI Psoriasis Area and Severity Score, $P B O$ placebo, $R Z B$ risankizumab

higher rate compared with the PASI score following treatment discontinuation. Despite the significant durability of patients' response to risankizumab following withdrawal, the deterioration of HRQL is more rapid. These findings align with previous studies, which show that, regardless of the reason for treatment gaps, treatment discontinuation negatively affects patients' HRQL, particularly those responsive to therapy. Results from a post hoc subanalysis of the double-blind, phase 3 REVEAL trial reported a twofold greater deterioration of HRQL measured by DLQI compared with PASI following withdrawal of adalimumab [30]. Although the results do not allow the identification of the cause for the disproportionate worsening of DLQI, previous research suggests that psychological impacts of disease worsening may be responsible for this pattern [30, 31]. Given the importance in identifying the impact of changes in HRQL to guide treatment optimization, additional studies are warranted to further assess the impact of treatment withdrawal among patients with psoriasis.

Methodologically, this study used several regression and machine learning model 
specifications based on patient characteristics (e.g., age, disease duration, treatment history) and well-established measures of disease severity to achieve robust performance in predicting DLQI scores and thus overcome the challenge of unmeasured scores. The cross-validation procedure helped protect against overfitting, while the additional model testing with IMMhance data at weeks 0,12 , and 16 indicated high outof-sample predictive ability. Results of the present study showed that the elastic net model achieved the best trade-off between predictive accuracy and model parsimony, highlighting the value of machine learning approaches in selecting relevant predictors among multiple highly correlated measures.

Overall, the findings from the machine learning procedure align with results from prior studies that analyzed determinants of HRQL as assessed using DLQI [32-38]. Several studies to date have reported a strong relationship between PASI and DLQI, in diverse patient populations and various settings worldwide [32-38]. Moreover, this is the first study to develop a model for out-of-sample prediction of DLQI based on a diverse set of patient and disease characteristics in psoriasis, and explains a higher proportion of the variation than a previous multivariate model [33]. Based on the models presented, sPGA was predictive of DLQI independently of PASI. This is consistent with previous research showing that, although sPGA and PASI correlate well, the correlation is weaker at the ends of the distribution, which indicates that the two measures may provide independent information about disease severity [39]. The residual predictive ability of the head subscore of PASI was also consistent with a prior study that demonstrated that patients with psoriasis with visible psoriatic lesions have significantly worse HRQL compared with those with nonvisible lesions [33].

Finally, the preferred model specification suggests that the relationship between PASI and DLQI is nonlinear, as indicated by the selection of the PASI squared terms as statistically significant predictors of DLQI scores in addition to the linear PASI total score term. This is consistent with a previous analysis of the relationship between disease severity as measured by PASI and HRQL as measured by EQ-5D-3L [40], which also found a nonlinear association between the two measures. In that analysis, increases in PASI close to zero were shown to have a larger impact on EQ-5D-3L compared with an equally sized increase in PASI at the higher end of the scale, as determined by the inclusion of a PASI squared term as a significant predictor of EQ-5D-3L.

\section{Limitations}

This study has several limitations. First, as with all trial-based analyses, the results may not be generalizable to a real-world population, as the study population comprised a group of patients selected on the basis of specific enrollment criteria and followed under well-controlled conditions. Second, despite the high predictive performance of the selected model, the exclusion of other variables not measured in the trial or unavailable at all time points may contribute to prediction error at the individual patient level. The model closely predicted aggregatelevel average DLQI scores during external validation, but does not fully explain heterogeneity in DLQI scores across different patients. Third, this study assumed that achieving a given level of clinical symptom control (as measured by PASI and sPGA scores) has the same HRQL benefits regardless of the specific treatment strategy that resulted in that symptom control. However, patients may experience different types and rates of adverse events depending on treatment, which could also contribute to heterogeneity in the HRQL benefits of different treatment strategies. Lastly, inherent limitations exist for the assessments of interest. For example, limitations with the DLQI include inadequate measurement of mild illness and differential item functioning by disease, age, and sex [41, 42], whereas limitations of PASI include poor sensitivity, nonlinear scale, lack of correspondence to clinical severity categories [43], and dependence on the experience level of the clinician performing the measurement [44]. Moreover, lower PASI values are less clinically meaningful, thus precluding valid comparisons across the entire spectrum of PASI scores [30]. 


\section{CONCLUSIONS}

Predicted DLQI scores revealed an association between risankizumab treatment withdrawal and HRQL deterioration, as evidenced by a progressive increase in the mean change in the predicted DLQI scores among patients who discontinued versus those who remained on treatment. The predicted HRQL deteriorated more rapidly than the PASI score, an objective measure of disease.

\section{ACKNOWLEDGEMENTS}

Funding. Sponsorship for this study and Rapid Service Fee were funded by AbbVie Inc. The study sponsor contributed to the design; participated in collection, analysis, and interpretation of data; and participated in writing, reviewing, and approval of the final version.

Authorship. All named authors meet the International Committee of Medical Journal Editors (ICMJE) criteria for authorship for this article, take responsibility for the integrity of the work as a whole, and have given their approval for this version to be published.

Authors' contributions. KAP, AMS, ND, and LP contributed to study conceptualization and design. ND, CC, and ELW performed or supported the formal analyses. All authors (KAP, AMS, ND, CC, ELW, and LP) contributed to the data interpretation, critically reviewed the manuscript, and approved the submitted manuscript version.

Medical writing, editorial, and other assistance. The authors would like to thank Gloria DeWalt from Analysis Group, Inc. for providing editorial assistance. Support for this assistance was provided by AbbVie Inc.

Disclosures. Kim A. Papp received honoraria or fees for serving on advisory boards, or as a speaker, or as a consultant, and grants as an investigator from AbbVie, Amgen, Astellas, Baxalta, Baxter, Boehringer Ingelheim, Bristol-
Myers Squibb, Celgene, Coherus, Dermira, Eli Lilly, Forward Pharma, Galderma, Genentech, GlaxoSmithKline, Janssen, Kyowa-Hakko Kirin, LEO Pharma, MedImmune, Merck-Serono, Merck Sharp \& Dohme, Novartis, Pfizer, Regeneron, Roche, Sanofi-Genzyme, Stiefel, Sun Pharma, Takeda, UCB, and Valeant. Ahmed M. Soliman is an employee of AbbVie, Inc. and owns stocks in AbbVie, Inc. Nicolae Done, Christopher Carley, and Esteban Lemus Wirtz are employees of Analysis Group, Inc., which received payment from AbbVie Inc. to conduct this research. Luis Puig received grants and personal fees from AbbVie during the conduct of the study; grants and personal fees from Almirall, Amgen, Boehringer Ingelheim, Celgene, Janssen, LEO Pharma, Lilly, Novartis, Pfizer, Regeneron, Sanofi, Roche, and UCB outside the submitted work; and personal fees from Sandoz, Merck-Serono, Merck Sharp \& Dohme, Mylan, and Samsung-Bioepis outside the submitted work

Compliance with Ethics Guidelines. This article is based on previously conducted studies and does not contain any new studies with human participants performed by any of the authors. The trials included in this study were approved by each site's institutional review board and was performed in accordance with the Helsinki Declaration of 1964, and its later amendments.

Prior presentation. Presented at the 2021 American Academy of Dermatology Virtual Meeting.

Data availability. AbbVie is committed to responsible data sharing regarding the clinical trials we sponsor. This includes access to anonymized, individual and trial-level data (analysis data sets), as well as other information (e.g., protocols and Clinical Study Reports), as long as the trials are not part of an ongoing or planned regulatory submission. This includes requests for clinical trial data for unlicensed products and indications. This clinical trial data can be requested by any qualified researchers who engage in rigorous, independent scientific research, and will be provided following review 
and approval of a research proposal and Statistical Analysis Plan (SAP) and execution of a Data Sharing Agreement (DSA). Data requests can be submitted at any time and the data will be accessible for 12 months, with possible extensions considered. For more information on the process, or to submit a request, visit the following link: https://www.abbvie.com/ourscience/clinical-trials/clinical-trials-data-andinformation-sharing/data-and-informationsharing-with-qualified-researchers.html.

Open Access. This article is licensed under a Creative Commons Attribution-NonCommercial 4.0 International License, which permits any non-commercial use, sharing, adaptation, distribution and reproduction in any medium or format, as long as you give appropriate credit to the original author(s) and the source, provide a link to the Creative Commons licence, and indicate if changes were made. The images or other third party material in this article are included in the article's Creative Commons licence, unless indicated otherwise in a credit line to the material. If material is not included in the article's Creative Commons licence and your intended use is not permitted by statutory regulation or exceeds the permitted use, you will need to obtain permission directly from the copyright holder. To view a copy of this licence, visit http://creativecommons.org/licenses/bync/4.0/.

\section{REFERENCES}

1. Conrad C, Gilliet M. Psoriasis: from pathogenesis to targeted therapies. Clin Rev Allergy Immunol. 2018;54(1):102-13.

2. Donigan JM, Pascoe VL, Kimball AB. Psoriasis and herpes simplex virus are highly stigmatizing compared with other common dermatologic conditions: a survey-based study. J Am Acad Dermatol. 2015;73(3):525-6.

3. de Korte J, Mombers FMC, Bos JD, Sprangers MAG. Quality of life in patients with psoriasis: a systematic literature review. J Investig Dermatol Symp Proc. 2004;9(2):140-7.
4. Founta O, Adamzik K, Tobin A-M, Kirby B, Hevey D. Psychological distress, alexithymia and alcohol misuse in patients with psoriasis: a cross-sectional study. J Clin Psychol Med Settings. 2019;26(2): 200-19.

5. Yeung H, Takeshita J, Mehta NN, Kimmel SE, Ogdie A, Margolis DJ, et al. Psoriasis severity and the prevalence of major medical comorbidity: a population-based study. JAMA Dermatol. 2013;149(10): 1173-9.

6. Koo J. Population-based epidemiologic study of psoriasis with emphasis on quality of life assessment. Dermatol Clin. 1996;14(3):485-96.

7. Michalek IM, Loring B, John SM. A systematic review of worldwide epidemiology of psoriasis. J Eur Acad Dermatol Venereol. 2017;31(2):205-12.

8. Singh S, Young P, Armstrong AW. An update on psoriasis and metabolic syndrome: a meta-analysis of observational studies. PLoS One. 2017;12(7): e0181039.

9. Gisondi P, Fostini AC, Fossa I, Girolomoni G, Targher G. Psoriasis and the metabolic syndrome. Clin Dermatol. 2018;36(1):21-8.

10. Levine D, Gottlieb A. Evaluation and management of psoriasis: an internist's guide. Med Clin North Am. 2009;93(6):1291-303.

11. Svoboda SA, Ghamrawi RI, Owusu DA, Feldman SR. Treatment goals in psoriasis: which outcomes matter most? Am J Clin Dermatol. 2020;21(4): 505-11.

12. Fredriksson T, Pettersson U. Severe psoriasis-oral therapy with a new retinoid. Dermatology. 1978;157(4):238-44.

13. CfMPfHUC EMA. Guideline on clinical investigation of medicinal products indicated or the treatment of psoriasis. London: EMEA; 2004.

14. Lebwohl MG, Bachelez H, Barker J, Girolomoni G, Kavanaugh A, Langley RG, et al. Patient perspectives in the management of psoriasis: results from the population-based Multinational Assessment of Psoriasis and Psoriatic Arthritis Survey. J Am Acad Dermatol. 2014;70(5):871-81-e1-30.

15. Takeshita J, Callis Duffin K, Shin DB, Krueger GG, Robertson $\mathrm{AD}$, Troxel $\mathrm{AB}$, et al. Patient-reported outcomes for psoriasis patients with clear versus almost clear skin in the clinical setting. J Am Acad Dermatol. 2014;71(4):633-41.

16. Finlay AY, Khan G. Dermatology Life Quality Index (DLQI) - a simple practical measure for routine clinical use. Clin Exp Dermatol. 1994;19(3):210-6. 
17. Li W, Ghamrawi R, Haidari W, Feldman SR. Risankizumab for the treatment of moderate to severe plaque psoriasis. Ann Pharmacother. 2020;54(4): 380-7.

18. Blauvelt A, Leonardi CL, Gooderham M, Papp KA, Philipp S, Wu JJ, et al. Efficacy and safety of continuous risankizumab therapy vs treatment withdrawal in patients with moderate to severe plaque psoriasis: a phase 3 randomized clinical trial. JAMA Dermatol. 2020;156(6):649-58.

19. Gordon KB, Strober B, Lebwohl M, Augustin M, Blauvelt A, Poulin Y, et al. Efficacy and safety of risankizumab in moderate-to-severe plaque psoriasis (UltIMMa-1 and UltIMMa-2): results from two double-blind, randomised, placebo-controlled and ustekinumab-controlled phase 3 trials. Lancet. 2018;392(10148):650-61.

20. Reich K, Gooderham M, Thaçi D, Crowley JJ, Ryan C, Krueger JG, et al. Risankizumab compared with adalimumab in patients with moderate-to-severe plaque psoriasis (IMMvent): a randomised, doubleblind, active-comparator-controlled phase 3 trial. Lancet. 2019;394(10198):576-86.

21. Risankizumab meets all primary endpoints reporting positive results in fourth pivotal phase 3 psoriasis study. Available at: https://news.abbvie.com/ news/risankizumab-meets-all-primary-endpointsreporting-positive-results-in-fourth-pivotal-phase3-psoriasis-study.htm [press release]. Accessed date 4 Dec 2017

22. Viswanathan HN, Chau D, Milmont CE, Yang W, Erondu N, Revicki DA, et al. Total skin clearance results in improvements in health-related quality of life and reduced symptom severity among patients with moderate to severe psoriasis. J Dermatol Treat. $2015 ; 26(3): 235-9$.

23. Hsu DY, Gniadecki R. Patient adherence to biologic agents in psoriasis. Dermatology. 2016;232(3): 326-33.

24. Mrowietz U, De Jong E, Kragballe K, Langley R, Nast A, Puig L, et al. A consensus report on appropriate treatment optimization and transitioning in the management of moderate-to-severe plaque psoriasis. J Eur Acad Dermatol Venereol. 2014;28(4): 438-53.

25. Daudén E, Puig L, Ferrándiz C, Sánchez-Carazo J, Hernanz-Hermosa J, Dermatology SPGotSAo, et al. Consensus document on the evaluation and treatment of moderate-to-severe psoriasis: Psoriasis Group of the Spanish Academy of Dermatology and Venereology. J Eur Acad Dermatol Venereol. 2016;30:1-18.
26. Doshi JA, Takeshita J, Pinto L, Li P, Yu X, Rao P, et al. Biologic therapy adherence, discontinuation, switching, and restarting among patients with psoriasis in the US Medicare population. J Am Acad Dermatol. 2016;74(6):1057-65 e4.

27. Bonafede $\mathrm{M}$, Johnson BH, Fox KM, Watson C, Gandra SR. Treatment patterns with etanercept and adalimumab for psoriatic diseases in a real-world setting. J Dermatol Treat. 2013;24(5):369-73.

28. Yeung H, Wan J, Van Voorhees AS, Callis Duffin K, Krueger GG, Kalb RE, et al. Patient-reported reasons for the discontinuation of commonly used treatments for moderate to severe psoriasis. J Am Acad Dermatol. 2013;68(1):64-72.

29. Belinchon I, Ramos JM, Carretero G, Ferrandiz C, Rivera R, Dauden E, et al. Adverse events associated with discontinuation of the biologics/classic systemic treatments for moderate-to-severe plaque psoriasis: data from the Spanish Biologics Registry. Biobadaderm J Eur Acad Dermatol Venereol. 2017;31(10):1700-8.

30. Poulin Y, Sheth P, Gu Y, Teixeira HD. Health-related quality of life worsens disproportionately to objective signs of psoriasis after withdrawal of adalimumab therapy. Dermatol Ther (Heidelb). 2014;4(1):33-42.

31. Ghajarzadeh M, Ghiasi M, Kheirkhah S. Associations between skin diseases and quality of life: a comparison of psoriasis, vitiligo, and alopecia areata. Acta Med Iran. 2012;1:511-5.

32. Daudén E, Pujol RM, Sánchez-Carazo JL, Toribio J, Vanaclocha F, Puig L, et al. Demographic characteristics and health-related quality of life of patients with moderate-to-severe psoriasis: the VACAP study. Actas Dermosifiliogr. 2013;104(9):807-14.

33. Herédi E, Rencz F, Balogh O, Gulácsi L, Herszényi K, Holló $\mathrm{P}$, et al. Exploring the relationship between EQ-5D, DLQI and PASI, and mapping EQ-5D utilities: a cross-sectional study in psoriasis from Hungary. Eur J Health Econ. 2014;15(1):111-9.

34. Palotai T, Szepetowski JC, Pec J, Arenberger P, Giurcaneanu C, Gyulai R, et al. A survey of disease severity, quality of life, and treatment patterns of biologically naive patients with psoriasis in central and eastern Europe. Acta Dermatovenerol Croat. 2010;18(3):1.

35. Hawro T, Zalewska A, Hawro M, Kaszuba A, Krolikowska M, Maurer M. Impact of psoriasis severity on family income and quality of life. J Eur Acad Dermatol Venereol. 2015;29(3):438-43.

36. Moradi M, Rencz F, Brodszky V, Moradi A, Balogh $\mathrm{O}$, Gulácsi L. Health status and quality of life in 
patients with psoriasis: an Iranian cross-sectional survey. Arch Iran Med. 2015;18(3):1.

37. Mabuchi T, Yamaoka H, Kojima T, Ikoma N, Akasaka E, Ozawa A. Psoriasis affects patient's quality of life more seriously in female than in male in Japan. Tokai J Exp Clin Med. 2012;37(3):84-8.

38. Tsai T-F, Ho J-C, Chen Y-J, Hsiao P-F, Lee W-R, Chi $\mathrm{C}-\mathrm{C}$, et al. Health-related quality of life among patients with moderate-to-severe plaque psoriasis in Taiwan. Dermatol Sin. 2018;36(4):190-5.

39. Robinson A, Kardos M, Kimball AB. Physician Global Assessment (PGA) and Psoriasis Area and Severity Index (PASI): why do both? A systematic analysis of randomized controlled trials of biologic agents for moderate to severe plaque psoriasis. J Am Acad Dermatol. 2012;66(3):369-75.

40. Geale K, Henriksson M, Schmitt-Egenolf M. How is disease severity associated with quality of life in psoriasis patients? Evidence from a longitudinal population-based study in Sweden. Health Qual Life Outcomes. 2017;15(1):151.
41. Bronsard V, Paul C, Prey S, Puzenat E, Gourraud PA, Aractingi S, et al. What are the best outcome measures for assessing quality of life in plaque type psoriasis? A systematic review of the literature. J Eur Acad Dermatol Venereol. 2010;24:17-22.

42. Twiss J, Meads DM, Preston EP, Crawford SR, McKenna SP. Can we rely on the Dermatology Life Quality Index as a measure of the impact of psoriasis or atopic dermatitis? J Invest Dermatol. 2012;132(1):76-84.

43. Berth-Jones J, Grotzinger K, Rainville C, Pham B, Huang J, Daly S, et al. A study examining inter- and intrarater reliability of three scales for measuring severity of psoriasis: Psoriasis Area and Severity Index, Physician's Global Assessment and Lattice System Physician's Global Assessment. Br J Dermatol. $2006 ; 155(4): 707-13$.

44. Langley RG, Ellis CN. Evaluating psoriasis with Psoriasis Area and Severity Index, Psoriasis Global Assessment, and Lattice System Physician's Global Assessment. J Am Acad Dermatol. 2004;51(4): 563-9. 